\title{
CME-Fragen bei CME.thieme.de
}

Viel Erfolg bei Ihrer CME-Teilnahme unter http://cme.thieme.de

Bitte informieren Sie sich über die genaue Gültigkeitsdauer unter http://cme.thieme.de

Sollten Sie Fragen zur Online-Teilnahme haben, unter http://cme.thieme.de/hilfe

finden Sie eine ausführliche Anleitung.

VNR 2760512017152370476

\section{Frage 1}

Welche Antwort ist richtig? Das Pringle-Manöver steht für:
A Bauchtuch-Tamponade zur Blutstillung bei Leberverlet- zungen
B vorübergehende Okklusion von A. und V. hepatica zur Blutungskontrolle
C vorübergehende Okklusion von A. hepatica und V. portae zur Blutungskontrolle
D Rekanalisation der V. umbilicalis
E Anschlingen der Lebervenen

\section{Frage 2}

Welche Antwort ist richtig? Das Chir-Net ist ein Kooperationsverbund...
A von Instituten für experimentelle Chirurgie
B für ein chirurgisches Studiennetzwerk
C für berufspolitische Fragen von Chirurgen
D für Tierversuche in der Chirurgie
E für molekulargenetische Forschung in der Chirurgie

\section{Frage 3}

Welcher Aspekt ist nicht zu den methodischen Limitationen der minimalinvasiven Chirurgie zu zählen?
A Die deutlich schlechtere Visualisierung
B Die Bergung von großen Organpakten/Resektaten
C Die ad-hoc-Kontrolle von Risikosituationen (z. B. Blutungen)
D Die sogenannte Viscerosynthese, d.h. Verbindung von Geweben, wie bei einer Anastomose
E Die suffiziente Exposition auch von schwer zugänglichen Arealen

\section{Frage 4}

Welche Aussage zum Einsatz und den Grenzen der minimalinvasiven Chirurgie trifft zu?
A Etwa $80 \%$ der viszeralchirurgischen Eingriffe werden heute minimalinvasiv durchgeführt
B Die Vorteile und teilweise Überlegenheit der minimalinva- siven Chirurgie konnte bis heute nicht belegt werden
C Die unzureichende Penetration minimalinvasiver Methoden liegt vor allem in methodischen Einschränkungen begründet
D Eine Verbesserung der minimalinvasiven Techniken ist auf absehbare Zeit nicht zu erwarten
E Die aktuellen Grenzen der minimalinvasiven Chirurgie

können durch den breiten Einsatz von NOTES (natural orifices transluminal surgery - Operieren über natürliche Körperöffnungen) vollständig kompensiert werden

\section{Frage 5}

Welche der folgenden Aussagen ist falsch? Limitationen der perioperativen Medizin und Akutschmerztherapie sind:
A Medizinische Limitationen
B Patientenbezogene Limitationen
C Fehlende wissenschaftliche Erkenntnisse
D Soziale Limitationen
E Konzeptionelle Limitationen

\section{Frage 6}

Welche der folgenden Aussagen zur Fast-track-Rehabilitation trifft nicht zu?
A Es handelt sich um ein bereits seit etwa 20 Jahren bekanntes Konzept.
B Lokal-chirurgische Komplikationen werden durch das Fast-track erheblich vermindert.
C Tradierte Verhaltensweisen verhindern die Einführung von Fast-track-Konzepten.
D Fast-track-Konzepte haben in randomisierten, kontrollierten Studien klinisch relevante Vorteile gezeigt.
E Es existieren zahlreiche Bezeichnungen für die optimierte perioperative Behandlung, wie Fast-track, ERAS, ESTREP etc., die aber identische Behandlungspfade beschreiben.

\section{Frage 7}

Welche der folgenden Aussagen ist richtig? Geriatrische Patienten...

A haben eine besonders stabile Homöostase, die nicht leicht gestört werden kann.

B weisen durch ihr hohes Alter per se schon ein erhöhtes Risiko auf.

C sollten postoperativ besonders zurückhaltend mobilisiert werden.

D können und sollten im Wesentlichen den gleichen Fast-trackRegimes unterzogen werden, wie jüngere Patienten.

E weisen aufgrund des verlangsamten Metabolismus eine besonders hohe Resistenz für unerwünschte Arzneimittelnebenwirkungen auf. 


\section{CME-Fragen bei CMIE.thiemede}

Fortsetzung ...

\section{Frage 8}

Welche der folgenden Patientengruppen stellt keine besondere Herausforderung im Rahmen der perioperativen Therapie und Akutschmerzbehandlung dar?
A Geriatrische Patienten
B Patienten mit fehlenden Kenntnissen der deutschen Sprache
C Patienten mit chronischen Schmerzen
D Patienten mit kognitiven Störungen
E Patienten, die intensiv Sport treiben

\section{Frage 9}

Welche Aussage zu Qualitäts-Dimensionen im Gesundheitswesen ist falsch?

A „Strukturqualität“ legt zugrunde, dass die richtigen Voraussetzungen an Ausstattung, Fähigkeiten und Fachkunde vorhanden sind.

B Unter „Prozessqualität“ versteht man, dass man das Richtige tut.

C „Ergebnisqualität“ setzt voraus, dass der erreichbare Zustand auch erreicht wird.

D Struktur- und Prozessqualität lassen sich vergleichsweise einfach messen und deren Optimierung kann rasch zu messbaren Verbesserungen führen.

E Eine Optimierung von Struktur- und Prozessqualität gewährleistet, dass in gleicher Weise auch die Ergebnisqualität zunimmt.

\section{Frage 10}

Welcher der folgenden Leistungsbereiche für operative Fächer ist aktuell nicht Gegenstand der externen Qualitätssicherung?
A Gynäkologische Operationen
B Herzschrittmacherversorgung inklusive Defibrillatoren
C Unfallchirurgie
D Hüft- und Knieendoprothesenversorgung
E Transplantationschirurgie 